\title{
Immune Thrombocytopenia: Antiplatelet Autoantibodies Inhibit Proplatelet Formation by Megakaryocytes and Impair Platelet Production in vitro José Perdomo
}

Haematology Research Unit, St George and Sutherland Clinical School, Faculty of Medicine, University of New South Wales, Sydney, NSW, Australia

Article Info

\section{Article Notes}

Received: September 3, 2018

Accepted: November 1, 2018

\section{*Correspondence:}

Dr. José Perdomo, Haematology Research Unit, St George and Sutherland Clinical School, Faculty of Medicine, University of New South Wales, Sydney, NSW, Australia; Telephone No: +61 2 91131915;

Email: j.perdomo@unsw.edu.au

C 2018 Perdomo J. This article is distributed under the terms of the Creative Commons Attribution 4.0 International License.
This commentary review considers the above publication, as invited by the Journal of Immunological Science. These data are already published and no additional data are provided. The paper deals with samples from 19 immune thrombocytopenia patients and was published in the journal Haematologica ${ }^{1}$.

Immune thrombocytopenia (ITP) is an autoimmune bleeding condition characterised by low platelet counts $\left(<100 \times 10^{9} / \mathrm{L}\right.$; reference count $150-400 \times 10^{9} / \mathrm{L}$ ) in the absence of other triggers that may cause thrombocytopenia ${ }^{2}$. Bleeding (petechiae, mucosal bleeding) is a common sign of ITP. There is a risk of internal bleeding and fatal haemorrhage has been reported ${ }^{3}$. ITP patients have reduced quality of life and decreased life expectancy ${ }^{4}$. Autoantibodies against abundant platelets antigens such as glycoproteins (GP) Ib/IX and IIb/ IIIa are found in ITP patients and contribute to platelet destruction. Platelet production by their precursor cells, megakaryocytes (MKs), is also decreased in ITP ${ }^{5}$. This is thought to occur via autoantibodymediated detrimental effects on MK differentiation and proliferation, which subsequently affect platelet production. These conclusions were drawn from observations of MKs cultured in the presence of ITP plasma ${ }^{6,7}$, antiplatelet monoclonal antibodies ${ }^{8}$ or serum from drug-induced ITP $^{9}$. However, a recent investigation in a murine passive model of ITP showed that most anti-platelet antibodies did not produce changes in $\mathrm{MK}$ numbers or morphology ${ }^{10}$. It should be noted that this work ${ }^{10}$ used monoclonal or polyclonal antibodies against mouse platelets and not ITP patient's-derived IgG. Nevertheless, as will be discussed below, the observations of this mouse model study are in agreement with our conclusions using ITP antibodies on human MKs in vitro ${ }^{1}$.

Treatment of ITP comprises several phases depending on severity and response. The goal of treatment is to decrease the risk of bleeding. The first line of therapy involves the use of corticosteroids or intravenous immunoglobulins. The objective of corticosteroid administration is to reduce antibody production ${ }^{11}$. In addition, steroid treatment also decreases platelet activation in responsive ITP patients ${ }^{12}$. Several mechanisms of action have been proposed for IVIg, including clearance of the autoantibodies mediated by $\mathrm{FcRn}^{13}$, ${ }^{14}$ and induction of the $\mathrm{Fc} \gamma$ inhibitory receptor ${ }^{15}$. The evidence for these mechanisms has been challenged (reviewed by ${ }^{16}$ ), and involvement of the Fc $\gamma$ RIIIa has been proposed ${ }^{16}$. These therapies can produce a rapid increase in platelet counts but do not produce a lasting response in most patients. Non-responsive patients can be 
treated with second line therapies such as splenectomy, which can be effective and long-lasting due to the removal of an organ that is involved in both platelet destruction and autoantibody production. Immunosuppressants such as anti CD20 (rituximab) ${ }^{17}$, cyclophosphamide ${ }^{18}$, cyclosporine or mycophenolate mofetil ${ }^{19}$, which inhibit $\mathrm{B}$ or T cells are also used as second line therapies. More recently thrombopoietin (TPO) receptor agonists (TPORAs) such as romiplostim and eltrombopag have entered clinical practice ${ }^{20}$. These agonists act by activating c-Mpl on precursor cells and promoting MK proliferation.

A well-recognised aspect of ITP is its heterogeneity, especially patients' response to treatment. TPO-RAs, for instance have a durable response mainly in nonsplenectomised patients ${ }^{21}$. In addition, long-lasting complete responses have been documented after discontinuation of TPO-RAs therapy ${ }^{22}$. Durable response is not a universal feature of these drugs and the nature of this immunomodulatory effect is yet to be described. The literature is still unclear about how these drugs increase platelet production. Leon et al showed an increase in MK proliferation (which is expected for TPO-RAs) leading to enhanced platelet production ${ }^{23}$, while other authors documented increased platelet numbers despite no changes in MK mass ${ }^{24}$.

Our study ${ }^{1}$ used MKs derived from cord blood CD $34^{+}$cells to address two aspects of ITP, i) the effect on ITP patients' serum and purified IgG on MK proliferation, maturation, apoptosis, proplatelet formation and platelet release; and ii) the activity of TPO-RAs on proplatelet formation in the presence of ITP autoantibodies. The findings of the study ${ }^{1}$ are summarised in Table 1 . The principal observation was that most ITP autoantibodies inhibited proplatelet formation and this was unrelated to effects on MK proliferation, differentiation, apoptosis or ploidy status. This implies that it is the final stage of megakaryopoiesis that is targeted by ITP antibodies, most likely by the effects of these antibodies on proplatelet structure ${ }^{25}$. Three serum samples affected MKs in culture but this was unrelated to the presence of anti-platelet antibodies and was most likely due to other components present in these sera such as TGF- $\beta 1$, PF4 or s-FAS ${ }^{1}$. In vivo, other mechanisms such as T cell imbalance are involved in ITP and may lead to reduction of MK production (reviewed $b^{26}{ }^{26}$, however in this case the experiments were performed in vitro in the absence of other cells.

Historically, it was thought that ITP was characterized by platelet destruction, but it is now apparent that insufficient platelet production is a substantial feature of ITP pathogenesis. Our work shows that IgG from most patients decreases proplatelet formation and consequently platelet production without evident effects on other MK features such ploidy, proliferation, cell size and apoptosis ${ }^{1}$. Damage to proplatelet structures by ITP antibodies in the absence of obvious apoptotic changes was also documented in a study by Lev at $a 2^{25}$, which was conducted mostly concurrently with our work and is also supported by observations of normal MK numbers and morphology in a mouse model of ITP $^{10}$. More recently, it was shown that thrombocytopenic patients (ITP and myelodysplastic syndrome) had reduced rather than increased MK apoptosis, which corroborates the in vitro observation of no significant apoptosis induction upon ITP antibody treatment.

TPO-RAs represent a relatively effective therapy for patients refractory to first line treatments. This in turn increases platelet production. Unexpectedly, MK proliferation has been observed in both responders and non-responders to TPO-RA administration ${ }^{27}$, suggesting that in some patients there is no direct correlation between MK proliferation and increase in platelet counts. Our work $^{1}$ offers an explanation by the surprizing finding that TPO-RAs not only promote MK proliferation but can also neutralize the inhibitory activity of some ITP antibodies, thus increasing the number of proplatelet-bearing MKs. In addition, the response to the TPO-RAs used in our study ${ }^{1}$ (romiplostim and eltrombopag) varied among patient samples, suggesting that some non-responders might benefit by switching TPO-RAs. This might become more pertinent once additional TPO-RAs such as avatrombopag ${ }^{28}$ become widely available. This study does not question the view that platelet destruction is also a key feature of ITP, as documented many decades ago ${ }^{29}$. In fact, several mechanisms, including phagocytosis of opsonised platelets by macrophages ${ }^{30}$, complement-mediated destruction ${ }^{31}$,

Table 1. Effect of ITP serum or IgG on cultured MKs

\begin{tabular}{|l|c|c|c|c|}
\hline & \multicolumn{4}{|c|}{ 19 ITP Patients $^{\mathrm{a}}$} \\
\hline Effect $^{\mathrm{b}}$ & Serum & IgG $^{\mathrm{c}}$ & $P$ value $^{\mathrm{e}}$ \\
\hline Decrease in Proplatelet formation & $8(42 \%)$ & $<$ value $^{\mathrm{e}}$ & $13(68 \%)$ & $<0.0001$ \\
\hline Reduction of MK proliferation & $3(15.7 \%)$ & 0.0001 & 0 & \\
\hline Reduction of MK maturation & $3(15.7 \%)$ & $<0.0001$ & 0 & \\
\hline Decrease in cell size & $3(15.7 \%)$ & $<0.0001$ & 0 & \\
\hline
\end{tabular}

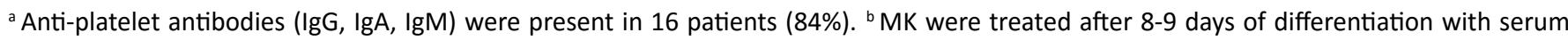
or total IgG at 1:10 dilution and evaluated 3-5 days later. Percentages shown are relative to control serum or control IgG ( $n=9$ ). ${ }^{c}$ Serum was obtained from coagulated blood and heat-inactivated at $56^{\circ} \mathrm{C}$ for $30 \mathrm{~min}$. ${ }^{\mathrm{d}}$ Total IgG was purified from ITP and normal sera with protein-G agarose beads, dialyzed and concentrated to $10 \mathrm{mg} / \mathrm{ml}$. ${ }^{e} P$ represents unpaired Student t-test. 
apoptosis and desialylation ${ }^{32}$ may account for platelet depletion in ITP. Liu et al reported that TPO-RAs may also inhibit platelet destruction by upregulation of inhibitory Fc $\gamma$ RII and suppression of activating Fc $\gamma$ receptors ${ }^{33}$. This expands the potential range of TPO-RA activities to MK proliferation, increase of pro-platelet formation per $\mathrm{MK}$ and inhibition of platelet destruction. In conclusion, it is evident that in both platelet destruction and production are contributors to the development of ITP. Our work shows that megakaryopoiesis is largely unaffected, but antibodymediated inhibition of proplatelet formation and platelet release is a regular feature of the pathogenesis of ITP.

\section{References}

1. Iraqi M, Perdomo J, Yan F, et al. Immune thrombocytopenia: antiplatelet autoantibodies inhibit proplatelet formation by megakaryocytes and impair platelet production in vitro. Haematologica. 2015.

2. Rodeghiero F, Stasi R, Gernsheimer T, et al. Standardization of terminology, definitions and outcome criteria in immune thrombocytopenic purpura of adults and children: report from an international working group. Blood. 2009; 113(11): 2386.

3. Cohen YC, Djulbegovic B, Shamai-Lubovitz O, et al. The bleeding risk and natural history of idiopathic thrombocytopenic purpura in patients with persistent low platelet counts. Arch Intern Med. 2000 160(11): 1630-8.

4. Shih A, Nazi I, Kelton JG, et al. Novel treatments for immune thrombocytopenia. Presse Med. 2014; 43(4 Pt 2): e87-95.

5. Perdomo J, Yan F, Chong BH. A megakaryocyte with no platelets: antiplatelet antibodies, apoptosis, and platelet production. Platelets. 2013; 24(2): 98-106.

6. Chang M, Nakagawa PA, Williams SA, et al. Immune thrombocytopenic purpura (ITP) plasma and purified ITP monoclonal autoantibodies inhibit megakaryocytopoiesis in vitro. Blood. 2003; 102(3): 887-95.

7. McMillan R, Wang L, Tomer A, et al. Suppression of in vitro megakaryocyte production by antiplatelet autoantibodies from adult patients with chronic ITP. Blood. 2004; 103(4): 1364-9.

8. Takahashi R, Sekine N, Nakatake T. Influence of monoclonal antiplatelet glycoprotein antibodies on in vitro human megakaryocyte colony formation and proplatelet formation. Blood. 1999; 93(6): 1951-8.

9. Perdomo J, Yan F, Ahmadi Z, et al. Quinine-induced thrombocytopenia: Drug-dependent GPIb/IX antibodies inhibit megakaryocyte and proplatelet production in vitro. Blood. 2011; 117(22): 5975-86.

10. Guo L, Kapur R, Aslam R, et al. Antiplatelet antibody-induced thrombocytopenia does not correlate with megakaryocyte abnormalities in murine immune thrombocytopenia. 2018(13653083 (Electronic)).

11. Mizutani H, Furubayashi T, Imai Y, et al. Mechanisms of corticosteroid action in immune thrombocytopenic purpura (ITP): experimental studies using ITP-prone mice, (NZW x BXSB) F1. Blood. 1992; 79(4): 942-7.

12. Bhoria P, Sharma S, Varma N, et al. Effect of steroids on the activation status of platelets in patients with Immune thrombocytopenia (ITP). Platelets. 2015; 26(2): 119-26.

13. Hansen RJ, Balthasar JP. Effects of intravenous immunoglobulin on platelet count and antiplatelet antibody disposition in a rat model of immune thrombocytopenia. Blood. 2002; 100 (6): 2087-93.

14. Sesarman A, Vidarsson G, Sitaru C. The neonatal Fc receptor as therapeutic target in IgG-mediated autoimmune diseases. Cell Mol Life Sci. 2010; 67(15): 2533-50.
15. Samuelsson A, Towers TL, Ravetch JV. Anti-inflammatory activity of IVIG mediated through the inhibitory Fc receptor. Science. 2001; 291(5503): 484-6.

16. Crow AR, Lazarus AH. Mechanistic properties of intravenous immunoglobulin in murine immune thrombocytopenia: support for FcgammaRIIB falls by the wayside. Semin Hematol. 2016; 53 Suppl 1: S20-2.

17. Arnold DM, Dentali F, Crowther MA, et al. Systematic review: efficacy and safety of rituximab for adults with idiopathic thrombocytopenic purpura. Ann Intern Med. 2007; 146(1): 25-33.

18. Reiner A, Gernsheimer T, Slichter SJ. Pulse cyclophosphamide therapy for refractory autoimmune thrombocytopenic purpura. Blood. 1995; 85(2): 351-8.

19. Taylor A, Neave L, Solanki S, et al. Mycophenolate mofetil therapy for severe immune thrombocytopenia. Br J Haematol. 2015; 171(4): 62530.

20. Siegal D, Crowther M, Cuker A. Thrombopoietin Receptor Agonists in Primary ITP. Semin Hematol. 2013; 50(0 1): S18-S21.

21. Kuter DJ, Bussel JB, Newland A, et al. Long-term treatment with romiplostim in patients with chronic immune thrombocytopenia: safety and efficacy. Br J Haematol. 2013; 161(3): 411-23.

22. Carpenedo M, Cantoni S, Coccini V, et al. Feasibility of romiplostim discontinuation in adult thrombopoietin-receptor agonist responsive patients with primary immune thrombocytopenia: an observational retrospective report in real life clinical practice. Hematol Rep. 2015; $7(1): 5673$

23. Leon C, Evert K, Dombrowski F, et al. Romiplostim administration shows reduced megakaryocyte response-capacity and increased myelofibrosis in a mouse model of MYH9-RD. Blood. 2012; 119(14): 3333-41.

24. Kuter DJ, Gernsheimer TB. Thrombopoietin and platelet production in chronic immune thrombocytopenia. Hematol Oncol Clin North Am. 2009; 23(6): 1193-211.

25. Lev PR, Grodzielski M, Goette NP, et al. Impaired proplatelet formation in immune thrombocytopenia: a novel mechanism contributing to decreased platelet count. Br J Haematol. 2014; 165(6): 854-64.

26. Ji X, Zhang L, Peng J, et al. T cell immune abnormalities in immune thrombocytopenia. J Hematol Oncol. 2014; 7: 72.

27. Barsam SJ, Psaila B, Forestier M, et al. Platelet production and platelet destruction: assessing mechanisms of treatment effect in immune thrombocytopenia. Blood. 2011; 117(21): 5723-32.

28. Bussel JB, Kuter DJ, Aledort LM, et al. A randomized trial of avatrombopag, an investigational thrombopoietin-receptor agonist, in persistent and chronic immune thrombocytopenia. Blood. 2014; 123(25): 3887

29. Harrington WJ, Minnich V, Hollingsworth JW, et al. Demonstration of a thrombocytopenic factor in the blood of patients with thrombocytopenic purpura. J Lab Clin Med. 1951; 38(1): 1-10.

30. Louwes H, Zeinali Lathori OA, Vellenga E, et al. Platelet kinetic studies in patients with idiopathic thrombocytopenic purpura. Am J Med. 1999; 106(4): 430-4

31. Kurata Y, Curd JG, Tamerius JD, et al. Platelet-associated complement in chronic ITP. Br J Haematol. 1985; 60(4): 723-33.

32. Li J, van der Wal DE, Zhu G, et al. Platelet Desialylation: A Novel Mechanism of Fc-Independent Platelet Clearance and a Potential Diagnostic Biomarker and Therapeutic Target in immune Thrombocytopenia. Blood. 2014; 124(21): 467.

33. Liu XG, Liu S, Feng Q et al. Thrombopoietin receptor agonists shift the balance of Fcgamma receptors toward inhibitory receptor IIb on monocytes in ITP. Blood. 2016; 128(6): 852-61. 